\title{
ChemComm
}

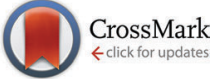

Cite this: Chem. Commun., 2016, 52,1843

Received 18th October 2015, Accepted 7th December 2015

DOI: $10.1039 / c 5 c c 08636 a$

www.rsc.org/chemcomm

\section{A molecular nanocap activated by superparamagnetic heating for externally stimulated cargo release $\dagger$}

\author{
B. Rühle, ${ }^{a}$ S. Datz, ${ }^{b}$ C. Argyo, ${ }^{b}$ T. Bein ${ }^{\text {b }}$ and J. I. Zink ${ }^{\star a}$
}

\begin{abstract}
A novel thermoresponsive snaptop for stimulated cargo release from superparamagnetic iron oxide core - mesoporous silica shell nanoparticles based on a [2+4] cycloreversion reaction (retro-Diels Alder reaction) is presented. The non-invasive external actuation through alternating magnetic fields makes this material a promising candidate for future applications in externally triggered drug delivery.
\end{abstract}

Mesoporous silica nanoparticles (MSNs) have attracted much attention as drug carriers in recent years. Using specific functionalization and design allows for controlled and targeted drug delivery from silica nanocarriers to specific target sites, such as cancer cells. ${ }^{1-5}$ However, there is still a great demand for spatial and temporal control of the release via external, non-invasive methods of actuation. Superparamagnetic iron oxide nanoparticles (SPIONs) can generate heat when exposed to an alternating magnetic field (AMF), ${ }^{6}$ and also act as contrast agents in $T_{2}$-weighted magnetic resonance imaging, making them an important tool in biomedical applications and theranostics. ${ }^{7-9}$ These characteristics can even be enhanced when using iron oxide nanoparticles that were doped with other metal ions that increase the magnetization, such as zinc and manganese ions. ${ }^{10}$

Combining both materials in a single, multifunctional core-shell nanostructure provides access to the advantages of both materials, i.e. a high loading capacity of various guests into the mesoporous silica shell, as well as localized superparamagnetic heating of the iron oxide core through an external AMF. ${ }^{11}$ This localized heating has the advantage of spatio-temporal control and activation using a deep tissue penetration stimulus. In consequence, decorating the pore openings of the MSNs with thermosensitive molecular gatekeepers that can act as valves and unblock the pores upon localized heating

\footnotetext{
${ }^{a}$ Department of Chemistry and Biochemistry, University of California Los Angeles, 607 Charles E. Young Drive East, Los Angeles, California 90095, USA. E-mail: zink@chem.ucla.edu

${ }^{b}$ Department of Chemistry and Center for NanoScience (CeNS),

University of Munich (LMU), Butenandtstraße 11 (E), 81377 München, Germany. E-mail: bein@lmu.de

$\dagger$ Electronic supplementary information (ESI) available: Detailed synthetic procedures, figures and a table. See DOI: 10.1039/c5cc08636a
}

is a promising concept for developing new, externally controlled, on-demand delivery systems for various cargo molecules. ${ }^{12}$

A particularly interesting concept for thermally triggered release of molecules is the cleavage of covalent chemical bonds. While this process often requires high temperatures, it is known that a concerted $[2+4]$ cycloaddition (Diels Alder reaction) and more importantly also the corresponding cycloreversion of maleimide derivatives with furan derivatives can proceed at mild temperatures. ${ }^{13-15}$ It has been demonstrated that the Diels Alder reaction of a malemide derivative with a furan derivative can be carried out on the surface of Stöber particles. ${ }^{16}$ Also, it was shown that the corresponding cycloreversion of Diels Alder adducts attached directly to the surface of superparamagnetic iron oxide or gold nanoparticles can be triggered by superparamagnetic heating ${ }^{17}$ or plasmonic heating, ${ }^{18,19}$ respectively. However, these examples require that the molecule that should be released features either a furan group or a maleimide group, which is not the case for many biologically or pharmaceutically relevant compounds. Moreover, in these examples each cycloreversion leads to the release of only one cargo molecule. A delivery system based on superparamagnetic iron oxide core - mesoporous silica shell nanoparticles (SPION@MSN) does not suffer from these limitations. The mesoporous silica shell features a high loading capacity for various cargo molecules without the requirement that they have specific functional groups. Additionally, when thermoresponsive gatekeepers are used to block the pore openings of the SPION@MSN and dissociate upon superparamagnetic heating, a single cycloreversion event will result in the release of multiple cargo molecules instead of just one.

While there are examples in the literature of thermoresponsive gatekeepers that can be operated through superparamagnetic heating, most of them are based on polymers or phase change materials ${ }^{20-26}$ and there are only few examples of small molecular nanovalves that block individual pores and can be controlled through AMFs. ${ }^{12}$ The advantage of these small molecular nanovalves is that the surface of the MSN can be further functionalized with additional functional groups such as targeting ligands, fluorescent probes, or even polymers such as PEG or PEI without affecting the operation of the valves. ${ }^{27}$ Moreover, because each of the 
molecular nanovalves is chemically identical, heterogeneities that occur during polymer capping due to the random coiling and entanglement of polymer chains or phase change materials are avoided, resulting in a more well-defined and homogeneous system.

In this work, we present a small molecular snaptop ${ }^{28-30}$ which blocks the pores of MSNs by supramolecular interactions between $\beta$-cyclodextrin and adamantane, ${ }^{31-34}$ while thermoresponsiveness is implemented by attaching the adamantane group to maleimidefunctionalized silica nanoparticles through a thermally reversible $[2+4]$ cycloaddition of a furan-modified linker (see Fig. 1a). Upon conventional or superparamagnetic heating, the system can undergo a cycloreversion, resulting in the dissociation of the furanadamantane- $\beta$-cyclodextrin moiety from the particle surface, which leads to pore unblocking and cargo release (see Fig. 1b).

Two different kinds of nanoparticles were prepared. Mesoporous silica nanoparticles without an iron oxide core (denoted MSN) that were used in the conventional heating experiments were synthesized under basic conditions with hexadecyltrimethylammonium bromide (CTAB) as the template according to a published procedure. ${ }^{35,36}$ The particles were grafted with maleimidopropyl triethoxysilane 1 (denoted MSN-Mal), which was synthesized by a two step procedure (see ESI $\dagger$ ). A co-condensation synthesis where maleimidopropyl triethoxysilane and tetraethylorthosilicate were reacted in a one-pot procedure was unsuccessful, probably due to hydrolysis of the maleimide under the basic conditions during MSN synthesis.

The zinc and manganese doped superparamagnetic iron oxide nanoparticles (SPIONs) with the formula $\left(\mathrm{Zn}_{0.4} \mathrm{Mn}_{0.6}\right) \mathrm{Fe}_{2} \mathrm{O}_{4}$ were chosen due to the larger magnetization of the doped SPIONs ( $\sim 200$ emu $\mathrm{g}^{-1}$, Fig. S10 $\left.\dagger\right)$ as compared to undoped SPIONs (typically 50-120 emu $\mathrm{g}^{-1}$ ), leading to an enhancement of superparamagnetic heating and MRI contrast. ${ }^{10}$ The doped SPION particles were synthesized by a thermal decomposition process of the metal salts in oleic acid/oleylamine/octyl ether according to literature (see also Fig. S10 $){ }^{10}$ TEM analysis shows that their approximate size is $8-10 \mathrm{~nm}$ (see Fig. $2 \mathrm{a}$ and S10 $\dagger$ ). In order to

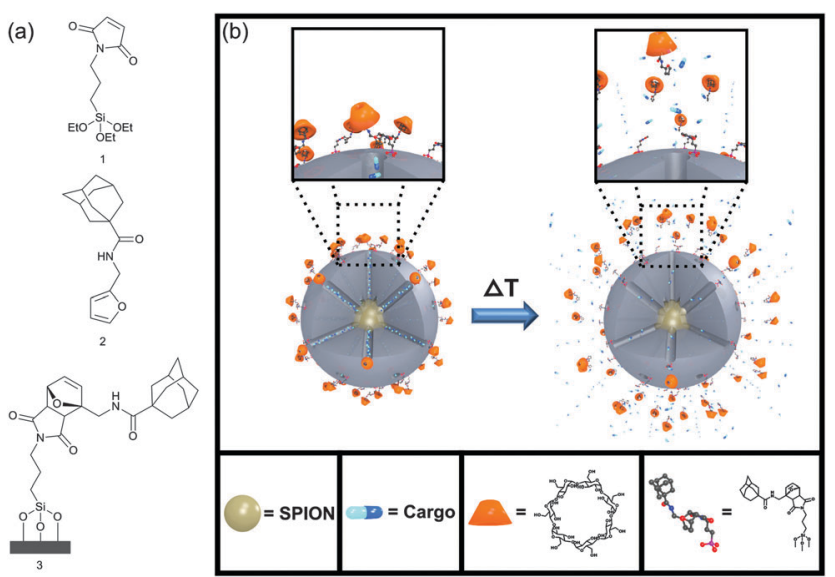

Fig. 1 (a) Chemical structures of the dienophile (1), the diene (2) and the Diels-Alder cycloaddition product (3). (b) Schematic representation of the operating principle of the thermoresponsive nanovalve. A change in temperature triggers a cycloreversion reaction which leads to a dissociation of the bulky gatekeepers from the silica surface and allows cargo molecules to diffuse out of the mesopores. coat them with mesoporous silica, the SPIONs were transferred to the aqueous phase by coating them with CTAC, followed by the addition of triethanolamine, and heating the mixture at $60{ }^{\circ} \mathrm{C}$. A stepwise addition of tetraethyl orthosilicate (TEOS) in small portions resulted in mesoporous silica shells in a layer-by-layer manner (see $\mathrm{ESI} \dagger$ for experimental details). After template removal from the sample by solvent extraction, the nanoparticles (denoted SPION@MSN) show a high BET surface area of $1034 \mathrm{~m}^{2} \mathrm{~g}^{-1}$, a total pore volume of $1.23 \mathrm{cc} \mathrm{g}^{-1}$, a mesopore volume (pore diameter < $6.5 \mathrm{~nm}$ ) of $0.81 \mathrm{cc} \mathrm{g}^{-1}$, and a pore diameter of $4.3 \mathrm{~nm}$ (see Fig. $2 \mathrm{~b}$ and Table S11 $\dagger$ ). The particles are about 70-80 $\mathrm{nm}$ in diameter (based on TEM observations, see Fig. 2a), have an effective hydrodynamic diameter of about $110 \mathrm{~nm}$ in water at $\mathrm{pH}=7$ (based on DLS measurements, see Fig. 2c) and a zeta potential at $\mathrm{pH}=7$ of $-21 \mathrm{mV}$ (Fig. 2d). After surfactant extraction, the particles were grafted with maleimidopropyl triethoxysilane 1 (the resulting sample is denoted SPION@MSN-Mal). The maleimide groups on the surfaces of samples MSN-Mal and SPION@MSN-Mal later act as the dienophile component in the Diels Alder reaction.

As the diene component, the adamantane-functionalized furan derivative 2 was synthesized in a one step procedure by acylation of furfurylamine with adamantane carbonyl chloride in dry dichloromethane in the presence of triethylamine (see $\mathrm{ESI} \uparrow$ for more details). The Diels Alder reaction of the maleimide groups attached to the silica surface of samples MSN-Mal and SPION@MSN-Mal and the furan-modified adamantane was then carried out in toluene for 3 days at $40{ }^{\circ} \mathrm{C}$, giving samples MSN-DA and SPION@MSN-DA, respectively.

The successful attachment of the maleimide and the formation of the Diels Alder cycloaddition product were confirmed by Fourier transform infrared spectroscopy (FTIR, Fig. 3), thermogravimetric analysis (TGA, Fig. S1 $\dagger$ ), and ${ }^{13} \mathrm{C}$ solid state NMR (ssNMR, Fig. S7 $\dagger$ ). The appearance of two bands in the IR spectrum due to carbonyl stretching vibrations at $\nu=1773 \mathrm{~cm}^{-1}$ and $\nu=1702 \mathrm{~cm}^{-1}$ indicate the presence of maleimide groups attached to the silica particles. This is also supported by newly emerging absorptions at $\nu=$ $1354 \mathrm{~cm}^{-1}$ (C-N-C stretching vibration), $\nu=845 \mathrm{~cm}^{-1}(\mathrm{C}-\mathrm{H}$ deformation vibration) and $\nu=697 \mathrm{~cm}^{-1}$ (maleimide ring deformation vibration). Additionally, two new characteristic amide absorptions (Amide I and Amide II) appear after the Diels Alder reaction at $\nu=1652 \mathrm{~cm}^{-1}$ and $\nu=1539 \mathrm{~cm}^{-1}$, respectively. At the same time the sharp band at $\nu=697 \mathrm{~cm}^{-1}$ disappears and a new, broader band at $\nu=702 \mathrm{~cm}^{-1}$ appears, indicating the successful cycloaddition of the furan derivative to the maleimide groups. Thermogravimetric analysis shows a mass loss of 13\%, 16\% and 23\% for samples SPION@MSN, SPION@MSN-Mal and SPION@MSN-DA after heating in air to $550{ }^{\circ} \mathrm{C}$, indicating the presence of organic molecules attached to the silica nanoparticles after the functionalization steps. The increase in weight loss from $16 \%$ to $23 \%$ after the Diels Alder reaction corresponds well to the theoretical $7 \%$ weight gain of the Diels-Alder addition product compared to the maleimidopropyl silane functionalized MSNs. ${ }^{13} \mathrm{C}$ ssNMR of sample MSN-DA shows new peaks at $\delta=177 \mathrm{ppm}, 138 \mathrm{ppm}, 128 \mathrm{ppm}, 90 \mathrm{ppm}, 79 \mathrm{ppm}$ and $48 \mathrm{ppm}$ (see green arrows in Fig. S7†) that can tentatively be assigned to the bicyclic cycloaddition product, while there are no peaks corresponding to the monocyclic ring carbon atoms of 
(a)

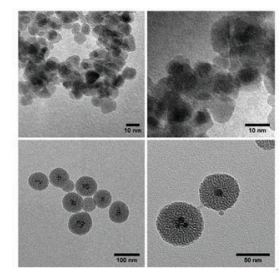

(c)

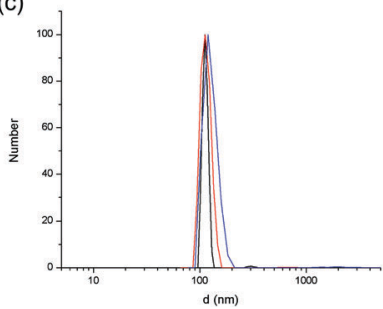

(b)

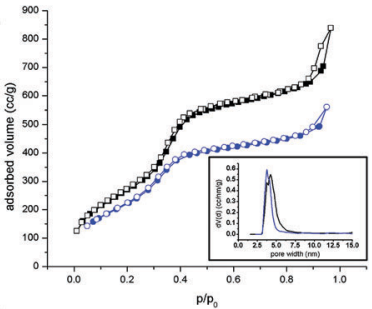

(d)

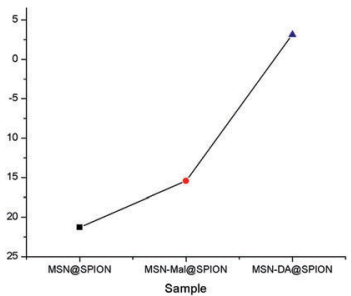

Fig. 2 (a) TEM images of zinc and manganese doped iron oxide nanoparticles ( $\left(\mathrm{Zn}_{0.4} \mathrm{Mn}_{0.6}\right) \mathrm{Fe}_{2} \mathrm{O}_{4}$; top) and SPION@MSN (bottom) at different magnifications; (b) nitrogen adsorption (filled symbols) and desorption (open symbols) isotherms of sample SPION@MSN (black squares) and SPION@MSN-DA (blue circles) (inset: NLDFT pore size distribution); (c) dynamic light scattering of samples SPIONaMSN (black), SPIONaMSN-Mal (red) and SPION@MSN-DA (blue); (d) zeta potential analysis at $\mathrm{pH}=7$ of samples SPION@MSN (black square), SPION@MSN-Mal (red circle) and SPION@MSN-DA (blue triangle; the line is a guide to the eye).

maleimide or furan; other signals from both components are however present. A strong shift of the monocyclic ring carbon signals is expected after forming the cycloaddition product, so these findings also indicate that the Diels-Alder reaction was successful and nearly quantitative.

Nitrogen sorption indicates a small loss of surface area, pore diameter and total pore volume after the functionalization steps, probably due to some extent of internal pore surface functionalization during silane grafting. However, with a remaining BET surface area of $859 \mathrm{~m}^{2} \mathrm{~g}^{-1}$, a pore diameter of $3.8 \mathrm{~nm}$ and a total pore volume of $0.82 \mathrm{cc} \mathrm{g}^{-1}$ the porosity of the functionalized material is still sufficient for its application as a carrier system (see Fig. 2b and Table S11 $\dagger$ ). As expected, dynamic light scattering does not show a significant change in hydrodynamic diameter of the particles

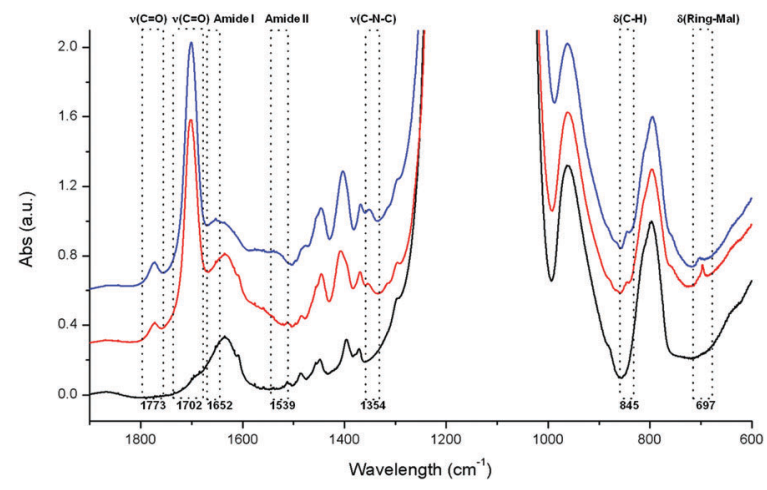

Fig. 3 IR data for samples SPION@MSN (black), SPION@MSN-Mal (red), and SPIONAMSN-DA (blue). The vibrations discussed in the text are highlighted by boxes. Peak assignments are based on literature data. ${ }^{37}$ For further information and raw spectral data see Fig. S2 in the ESI. $\dagger$

(approximately 110-120 $\mathrm{nm}$ ) after any of the modification steps (see Fig. 2c). The zeta potential at $\mathrm{pH}=7$ changes from $-21 \mathrm{mV}$ for sample SPION@MSN to $-\mathbf{1 5} \mathrm{mV}$ for sample SPION@MSN-Mal and then to an almost neutral value of $+3 \mathrm{mV}$ for sample SPION@ MSN-DA (see Fig. 2d). This can be explained by the fact that after surface functionalization with maleimide and especially with the hydrophobic adamantane-functionalized furan derivative, the negatively charged silanol groups on the surface of the silica nanoparticles are shielded by the uncharged organic moieties.

After attaching the adamantane-functionalized furan to the silica surface, fluorescein cargo was loaded into the mesoporous silica nanoparticles by soaking them in a $1 \mathrm{mM}$ aqueous solution overnight. The pore openings were then blocked by a bulky $\beta$-cyclodextrin moiety, which was bound to the adamantane groups through supramolecular interactions (samples MSN-CD and SPION@ MSN-CD). Carrying out the cycloaddition first and sealing the pores with a bulky gatekeeper at a later stage has the advantage that the experimental conditions for the loading step can be chosen independently from the reaction conditions for the cycloaddition reaction (i.e., organic solvents such as toluene, 3 days, $40{ }^{\circ} \mathrm{C}$ ) that might be incompatible with some cargo molecules.

In order to confirm that the intended cycloreversion and pore unblocking can be triggered by external heating, fluorescein release from sample MSN-CD was monitored at room temperature, $37{ }^{\circ} \mathrm{C}$ and $65{ }^{\circ} \mathrm{C}$, respectively. $0.5 \mathrm{mg}$ of $\mathbf{M S N}-\mathbf{C D}$ were placed in a reservoir that was separated from an aqueous solution inside a fluorescence cuvette by a $14 \mathrm{kDa}$ MWCO dialysis membrane, which is permeable to fluorescein but impermeable to the silica nanoparticles. The fluorescence intensity of fluorescein released into the cuvette was monitored over time. The temperature was adjusted externally by using a temperature-controlled cuvette holder, and the temperature dependence of fluorescein emission was corrected with the help of calibration curves obtained separately (see Fig. S8 and the ESI $\dagger$ for more details). The data show that there is almost no leakage at room temperature and $37{ }^{\circ} \mathrm{C}$, but a strong increase in fluorescein release upon heating from $37{ }^{\circ} \mathrm{C}$ to $65{ }^{\circ} \mathrm{C}$ (see Fig. 4a).

Encouraged by these findings and by the fact that a similar temperature increase should also be feasible through superparamagnetic heating of iron oxide core - silica shell nanoparticles, ${ }^{11}$ a similar release experiment was performed with sample SPION@MSN-CD in order to confirm that the release can also be triggered by superparamagnetic heating. After monitoring the release every $15 \mathrm{~min}$ for $1 \mathrm{~h}$ at room temperature, the sample was exposed to an AMF in a five-turn copper coil $(5 \mathrm{~cm}$ height and diameter) at a power of $5 \mathrm{~kW}$ and a frequency of $370 \mathrm{kHz}$ for $30 \mathrm{~min}$, followed again by monitoring the release at room temperature (the bulk solution temperature increased from room temperature to approx. $38^{\circ} \mathrm{C}$ directly after the AMF exposure, but cooled back down to room temperature before the next AMF cycle). In total, five such heating/monitoring cycles were performed (see Fig. 4b). The observed rise in fluorescein fluorescence indicates that a displacement of the $\beta$-cyclodextrin caps can also be triggered externally by local heating by an AMF. To exclude the possibility that the observed increase in fluorescein release is merely caused by bulk heating of the solution rather than localized superparamagnetic heating of SPION cores inside the mesoporous silica nanoparticles, a similar 

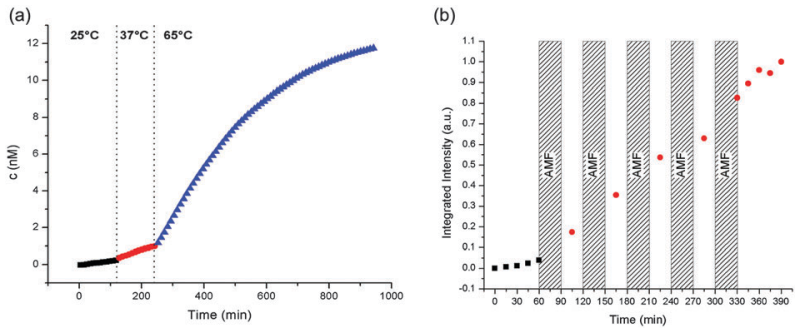

Fig. 4 Release of the fluorescein cargo from samples (a) MSN-CD and (b) SPIONaMSN-CD through (a) bulk solvent heating and (b) local superparamagnetic heating

release experiment was carried out, but the sample was kept in an ice bath at $0{ }^{\circ} \mathrm{C}$ at all times (see Fig. $\mathrm{S} 9 \dagger$ ). Under these conditions, the bulk solution temperature stays unchanged, and the heat generation is confined to the core-shell nanoparticles. As expected, the fluorescence intensity still rises after AMF exposure, indicating that indeed localized superparamagnetic heating triggers the release.

In conclusion, we have demonstrated that a thermally reversible cycloaddition reaction can be used to construct a molecular snaptop that can trap cargo inside the pores of mesoporous silica nanoparticles. The cycloreversion can be triggered by an externally applied AMF resulting in local particle heating and thus in the detachment of the cap from the pore openings and cargo release. This new concept of a molecular nanocap based on a retro-Diels Alder reaction activated through superparamagnetic heating adds to the toolbox of externally controllable, thermally triggered nanovalves. We envision that changing the electronic properties of the diene and dienophile component in the Diels Alder reaction should allow for further finetuning of the release properties of such nanovalves. Actuation through an AMF has the advantage of deep tissue penetration and non-invasiveness, making these nanovalves interesting candidates for future applications in drug delivery.

The authors thank the Defense Threat Reduction Agency (Grant HDTRA1-13-1-0046) and the Deutsche Forschungsgemeinschaft (NIM Excellence Cluster and SFB 1032) for financial support. We thank Dr Wen-Yen Huang for 2D NMR data acquisition.

\section{Notes and references}

1 C. Argyo, V. Weiss, C. Bräuchle and T. Bein, Chem. Mater., 2014, 26, 435-451.

2 D. Tarn, C. E. Ashley, M. Xue, E. C. Carnes, J. I. Zink and C. J. Brinker, Acc. Chem. Res., 2013, 46, 792-801.

3 S.-H. Wu, C.-Y. Mou and H.-P. Lin, Chem. Soc. Rev., 2013, 42, 3862-3875.

4 Z. Li, J. C. Barnes, A. Bosoy, J. F. Stoddart and J. I. Zink, Chem. Soc. Rev., 2012, 41, 2590-2605.
5 M. W. Ambrogio, C. R. Thomas, Y.-L. Zhao, J. I. Zink and J. F. Stoddart, Acc. Chem. Res., 2011, 44, 903-913.

6 R. E. Rosensweig, Proc. 9th Int. Conf. Magn. Fluids, 2002, 252, 370-374.

7 Y. Wang and H. Gu, Adv. Mater., 2015, 27, 576-585.

8 S. Laurent, D. Forge, M. Port, A. Roch, C. Robic, L. Vander Elst and R. N. Muller, Chem. Rev., 2008, 108, 2064-2110.

9 Q. A. Pankhurst, J. Connolly, S. K. Jones and J. Dobson, J. Phys. Appl. Phys., 2003, 36, R167.

10 J. Jang, H. Nah, J.-H. Lee, S. H. Moon, M. G. Kim and J. Cheon, Angew. Chem., Int. Ed., 2009, 48, 1234-1238.

11 J. Dong and J. I. Zink, ACS Nano, 2014, 8, 5199-5207.

12 C. R. Thomas, D. P. Ferris, J.-H. Lee, E. Choi, M. H. Cho, E. S. Kim, J. F. Stoddart, J.-S. Shin, J. Cheon and J. I. Zink, J. Am. Chem. Soc., 2010, 132, 10623-10625.

13 N. Kuramoto, K. Hayashi and K. Nagai, J. Polym. Sci. Part Polym. Chem., 1994, 32, 2501-2504.

14 B. Rickborn, Organic Reactions, John Wiley \& Sons, Inc., 2004.

15 A. M. Peterson, R. E. Jensen and G. R. Palmese, ACS Appl. Mater. Interfaces, 2010, 2, 1141-1149.

16 T. Engel and G. Kickelbick, Chem. Mater., 2013, 25, 149-157.

17 T. T. T. N'Guyen, H. T. T. Duong, J. Basuki, V. Montembault, S. Pascual, C. Guibert, J. Fresnais, C. Boyer, M. R. Whittaker, T. P. Davis and L. Fontaine, Angew. Chem., Int. Ed., 2013, 52, 14152-14156.

18 A. B. S. Bakhtiari, D. Hsiao, G. Jin, B. D. Gates and N. R. Branda, Angew. Chem., Int. Ed., 2009, 48, 4166-4169.

19 S. Yamashita, H. Fukushima, Y. Niidome, T. Mori, Y. Katayama and T. Niidome, Langmuir, 2011, 27, 14621-14626.

20 T.-Y. Liu, S.-H. Hu, D.-M. Liu, S.-Y. Chen and I.-W. Chen, Nano Today, 2009, 4, 52-65.

21 I. Y. Galaev and B. Mattiasson, Enzyme Microb. Technol, 1993, 15, 354-366.

22 C. Liu, J. Guo, W. Yang, J. Hu, C. Wang and S. Fu, J.Mater. Chem., 2009, 19, 4764-4770.

23 A. Baeza, E. Guisasola, E. Ruiz-Hernández and M. Vallet-Regí, Chem. Mater., 2012, 24, 517-524.

24 L. Dong, H. Peng, S. Wang, Z. Zhang, J. Li, F. Ai, Q. Zhao, M. Luo, H. Xiong and L. Chen, J. Appl. Polym. Sci., 2014, 131, 40477.

25 W. Guo, C. Yang, H. Lin and F. Qu, Dalton Trans., 2014, 43, 18056-18065.

26 R. C. S. Azevedo, R. G. Sousa, W. A. A. Macedo and E. M. B. Sousa, J. Sol-Gel Sci. Technol., 2014, 72, 208-218.

27 J. Dong, M. Xue and J. I. Zink, Nanoscale, 2013, 5, 10300-10306.

28 K. Patel, S. Angelos, W. R. Dichtel, A. Coskun, Y.-W. Yang, J. I. Zink and J. F. Stoddart, J. Am. Chem. Soc., 2008, 130, 2382-2383.

29 M. W. Ambrogio, T. A. Pecorelli, K. Patel, N. M. Khashab, A. Trabolsi, H. A. Khatib, Y. Y. Botros, J. I. Zink and J. F. Stoddart, Org. Lett., 2010, 12, 3304-3307.

30 T. M. Guardado-Alvarez, L. Sudha Devi, M. M. Russell, B. J. Schwartz and J. I. Zink, J. Am. Chem. Soc., 2013, 135, 14000-14003.

31 W. C. Cromwell, K. Bystrom and M. R. Eftink, J. Phys. Chem., 1985, 89, 326-332.

32 M. R. Eftink, M. L. Andy, K. Bystrom, H. D. Perlmutter and D. S. Kristol, J. Am. Chem. Soc., 1989, 111, 6765-6772.

33 D. Harries, D. C. Rau and V. A. Parsegian, J. Am. Chem. Soc., 2005, 127, 2184-2190.

34 M. Xue, D. Cao, J. F. Stoddart and J. I. Zink, Nanoscale, 2012, 4, 7569-7574.

35 H. Meng, M. Xue, T. Xia, Y.-L. Zhao, F. Tamanoi, J. F. Stoddart, J. I. Zink and A. E. Nel, J. Am. Chem. Soc., 2010, 132, 12690-12697.

36 J. Lu, M. Liong, J. I. Zink and F. Tamanoi, Small, 2007, 3, 1341-1346.

37 E. C. Aguiar, J. B. P. da Silva and M. N. Ramos, Mol. Spectrosc. Mol. Struct., 2011, 993, 431-434. 